\title{
An interference-reduction theory of the effects of ethanol on conflict behavior
}

\author{
JUDSON S. BROWN, JAMES G. MANSFIELD, and ARLIE J. SKURDAL \\ University of Oregon Health Sciences Center, Portland, Oregon 97201
}

\begin{abstract}
The classic tension-reduction theory of the effects of ethanol on approach-avoidance conflict behavior involves the assumption that the drug reduces conflict by selectively weakening fear-motivated avoidance but not approach. An alternative interpretation in which alcohol is seen as reducing the capacity of the weaker of any two competitive tendencies, whether inhibitory or excitatory, to interfere with the stronger is proposed here. Data from studies of isolated avoidance gradients and from experiments on approach-avoidance and avoidanceavoidance conflicts which support an interference-reduction rather than a tension-reduction theory are reported.
\end{abstract}

Although the tension-reduction theory of the effects of ethanol on behavior has been supported by the results of some experiments on approachavoidance conflict (e.g., Conger, 1951; Freed, 1967), the failure of other attempts to confirm it has raised serious questions as to its general utility (Cappell \& Herman, 1972). Perhaps it is too soon to abandon the hypothesis entirely, but it is not too early to consider alternative conceptualizations. One such possibility, to be explored here, is that in any situation in which at least two incompatible reactive tendencies are simultaneously instigated, ethanol reduces the capacity of the weaker to interfere with the stronger. As a result, ethanol tends to increase the relative superiority of any initially stronger tendency. In what follows, we shall examine the applicability of this interference-reduction conception to paradigms that generate strongly competitive reaction tendencies, namely, approach-avoidance and avoidance-avoidance conflict situations. Since the former provide the best support for the tensionreduction view, it is appropriate to begin by evaluating the applicability of our alternative conception to them.

\section{APPROACH-AVOIDANCE CONFLICT UNDER ETHANOL}

Conger's (1951) observation, that inebriated rats approach more closely than do sober ones to a place

This work was supported in part by Training Grant AA07074 from the National Institute of Alcohol Abuse and Alcoholism and by Research Grant AA01229 from the same agency. The second author is now at the Alcoholism and Drug Abuse Institute, University of Washington, Seattle, Washington. The authors have profited greatly from frequent discussions of these materials with Christopher L. Cunningham, and are deeply indebted to Craig Lee for his help in constructing equipment, in running subjects, and in analyzing data.

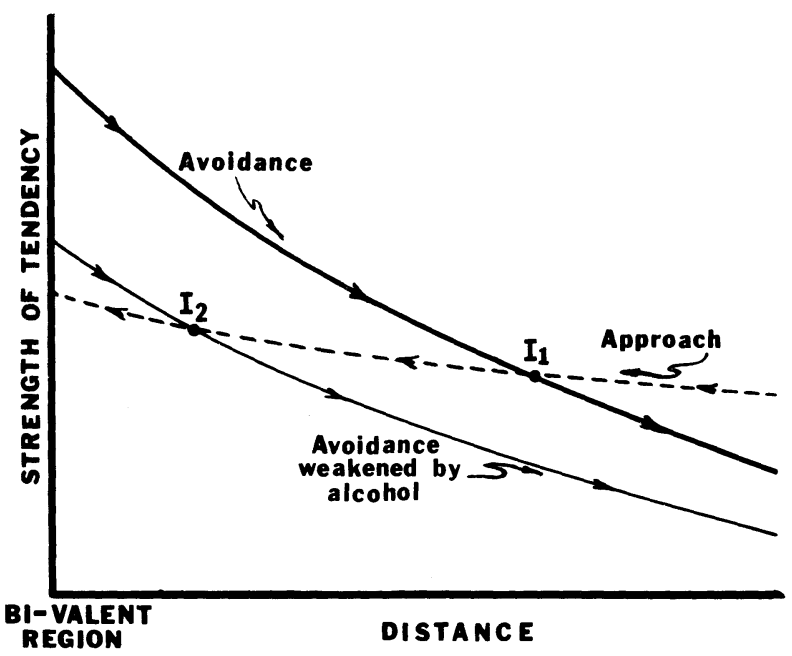

Figure 1. Hypothetical spatial gradients in the strengths of tendencies to approach toward (dashed line) and retreat from (heavy solid line) a place where both positive and negative reinforcers have been encountered. The thin solid line represents a possible effect of ethanol on the avoidance tendency and, in combination with the approach tendency, a resulting shift of the gradients' intersection from $I_{1}$ to $I_{2}$.

where both rewards and punishments have been administered, has long been taken as evidence for the tension-reduction hypothesis. The spatial gradients in Figure 1 summarize the central theoretical suppositions underlying his investigation, as well as the tension-reduction interpretation of his results. The heavy solid line represents the punishment-produced tendency to withdraw from the bivalent region at the left, and the dashed line is the reward-induced tendency to approach it. Both gradients represent tendencies of sober animals. Conger maintained that the avoidance tendency, but not the approach, was potentiated by a conditioned fear drive (tension) and that ethanol would weaken fear but not hunger. 
Consequently, the avordance gradient under ethanol would be lowered and flattened (light solid line) and its intersection with the unaltered approach gradient would shift in a goalward direction, as from $I_{1}$ to $\mathrm{I}_{2}$ in Figure 1. According to traditional conflict theory (Brown, 1957; Hull, 1938; Miller, 1944), approach and avoidance tendencies summate algebraically, and locomotion occurs in either direction when the net difference exceeds some threshold value. Movement ceases, therefore, when the intersection of the gradients is reached. Since intersection $I_{2}$ is closer to the bivalent region than $I_{1}$, it follows that the organism, when inebriated, and hence less fearful or tense, should approach the goal more closely than when sober. This was, indeed, the result Conger (1951) obtained. However, attempts by other investigators to confirm his findings have produced equivocal results (Barry \& Miller, 1962; Freed, 1968a, 1968b; Miller, 1956; Miller \& Barry, 1960; Smart, 1965).

Although Conger assumed that ethanol selectively attenuates avoidance by diminishing fear, in our view, it is the weaker tendency, whether to avoid or approach and whether excitatory or inhibitory, that is weakened by the drug. If overt behavior is determined by the net difference between the strengths of competing instigators, the capacity of the stronger to control the action should be heightened by ethanol. When approach is stronger than avoidance, as at the right side of Figure 1, the drug should increase the difference favoring approach; and when avoidance is the stronger, as at the left of $I_{1}$ in Figure 1, ethanol should increase the superiority of the avoidance tendency. Avoidance is weakened relative to approach in the first case, and approach is weakened relative to avoidance in the second. Both a fear-reduction hypothesis and our interference-reduction hypothesis thus predict that when animals are placed at the right of $I_{1}$ they will approach closer to the bivalent region when under the influence of alcohol than when not drugged. However, if animals are placed at the left of $I_{1}$, the first hypothesis predicts less extensive withdrawal under ethanol, whereas the second predicts more extensive withdrawal.

\section{Ethanol and the Isolated Avoidance Gradient}

Some of the early attempts to validate experimentally the fear-attenuation notion focused on ethanol's impact on avoidance tendencies alone. The fear-reduction hypothesis predicts that if an avoidance gradient is measured under both drug and control conditions it should be lower under the drug even in the absence of competitive tendencies. In our view, however, moderate doses of ethanol should have no effect under such conditions; ethanol should affect performance only when two or more tendencies to perform incompatible movements are simultaneously aroused. Tests of the effects of ethanol on un- opposed avoidance tendencies (Broadhurst \& Wallgren, 1964; Chittel \& Sheth, 1963; Harris, Picolino, Roback, \& Sommer, 1964) have indicated that the drug weakens avoidance behavior only at doses so high as to produce clear motor impairment. Because the present proposal suggests no effects of ethanol on avoidance gradients alone, these results can reasonably be attributed to simple motor effects. But unless the dose-response curve for motor effects is assumed to be identical to the dose-response curve for fear-attenuation effects, these results cannot be handled by a tension-reduction view. Concurrent tests of ethanol's impact on avoidance and approach tendencies (Barry \& Miller, 1965; Moskowitz \& Asato, 1966) have revealed some depression by alcohol of each tendency, but no differential effects. Again, the depression could be due to nonspecific effects of the drug. Finally, instances in which alcohol appears to facilitate avoidance behavior (Reynolds \& Van Sommers, 1960) can be encompassed by the present hypothesis by invoking the presence of subordinate interfering response tendencies.

Avoidance gradients such as those in Figure 1 are generally assumed to reflect the strengths of response tendencies in simple avoidance situations, as well as in conflict-generating conditions. However, avoidancelearning paradigms yield little information as to the overall form of the gradients, since measurements are typically taken at only one distance from the goal or represent average performance levels across the range of the gradient. In an attempt to determine more exactly how ethanol affects avoidance tendencies per se, we have conducted two very similar experiments (Brown \& Lee, Note 1) in which isolated avoidance speed gradients have been measured in their entirety under both drug and placebo conditions.

In each of these studies, 10 female albino rats (from Carworth Farms and Holtzman, Inc., in the first and second studies, respectively) were maintained on ad-lib food and water and were trained to escape from an electrified portion ( $46 \mathrm{~cm}$ long) at one end of a straight runway $(305 \mathrm{~cm}$ long, $12 \mathrm{~cm}$ wide). Shock $(.6 \mathrm{~mA}, 60 \mathrm{~Hz})$ was administered through the grid floor. After two such trials on each of 2 days, half the animals were injected with ethanol $(1.2 \mathrm{~g} / \mathrm{kg}$, ip) and half, with physiological saline. Fifteen minutes later, they were given 15 shock-free avoidance trials, on each of which they were dropped into the end of the runway where they had been shocked and then allowed to run freely toward the opposite end. Times taken to traverse each $30.5-\mathrm{cm}$ segment of the alley were recorded to the nearest $.02 \mathrm{sec}$ by means of infrared light beams, photocells, and associated devices. Segment speeds $(\mathrm{cm} / \mathrm{sec})$ were calculated from segment times.

Speeds measured over the first five test trials in 
these experiments are shown in Figures 2 and 3. Speed is assumed to reflect the strength of the response tendency in all segments save the first, where it is confounded by an acceleration component. Because of this, all analyses reported below involved data from Segments 2 through 10 only, although the same conclusions were reached from analyses that included Segment 1 data. Moreover, data obtained on the last 10 trials (presented later) were excluded here to minimize extinction effects.

In Experiment 1, the gradient was slightly lower under ethanol than under saline, but in Experiment 2, the relation was reversed. In neither case, however, were the main effect of ethanol $[\mathrm{F}(1,8)=1.95$, $\mathrm{p}>.05$, and $\mathrm{F}(1,8)=1.15, \mathrm{p}>.05$, respectively] and the interactions of drug treatment with alley segment $[F(7,56)=.07, p>.05$, and $F(7,56)<1.0]$ significant. The main effect of alley segment (distance from the shock zone) was significant $[F(7,56)$ $=29.8, \mathrm{p}<.01$, and $\mathrm{F}(7,56)=25.9, \mathrm{p}<.01]$ in both studies. Incidentally, the shapes of the saline gradients correspond closely to those of gradients reported by Somiya (1961).

The early-trial speed data from these two experiments thus fail to show that ethanol decreased the height and/or slope of the isolated avoidance gradient. But, given this result, why do inebriated animals approach closer to the bivalent region than do sober ones? Why does ethanol appear to increase the net approach tendency, as in Conger's experiment? Part of the answer may lie in a lack of correspondence between running speed and strength of pull, although methodological comparisons of these measures are unavailable. On the theory proposed here, ethanol brings about closer goal approach in the conflict situation not because it weakens avoidance by weakening fear, but because it weakens

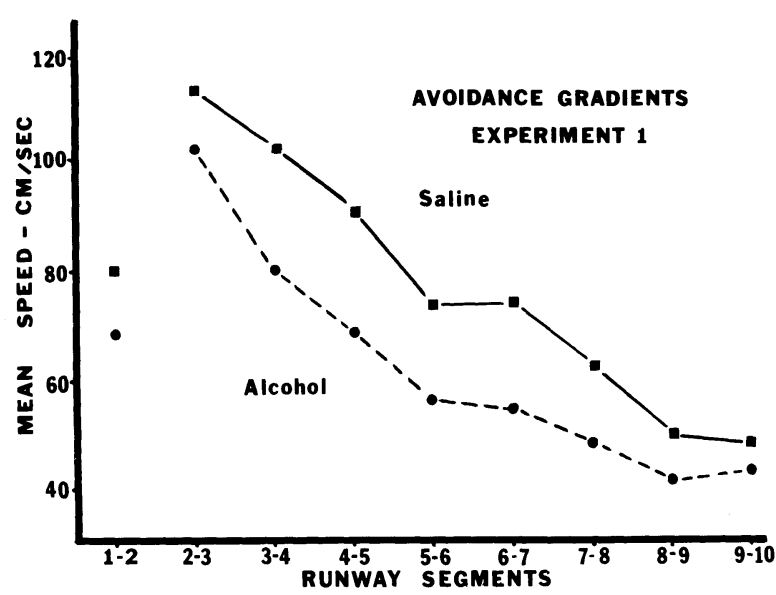

Figure 2. Mean shock-free avoidance speeds over five trials for saline-control and alcohol-treated rats as measured in each of $\mathbf{1 0}$ segments of a straight runway.

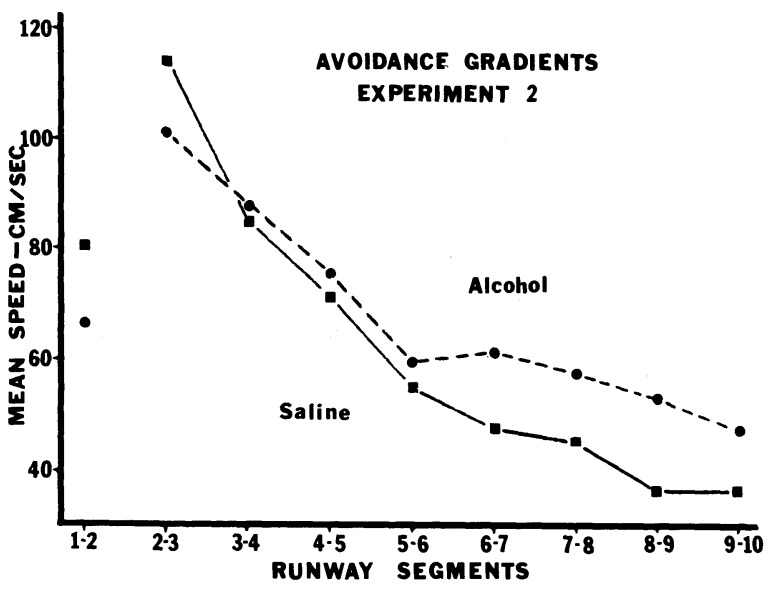

Figure 3. Mean avoidance running speeds in a straight alley averaged over five trials. Injections of alcohol and of saline were given shortly before the avoidance test trials.

whichever competitive tendency is already the weaker. When avoidance is very strong and is not opposed by competitive tendencies of any appreciable magnitude, as in the two studies just described, the drug should have little effect.

When all of the 15 nonreinforced test trials of the first experiment were included in an overall analysis (Treatments by Blocks by Alley Segments), the interaction of drug treatments with alley segment was significant $[F(7,56)=2.38, p<.05]$, suggesting that the drug had altered the slopes of the gradients. Graphic plots revealed that this result was due largely to increases in the speeds of the drugged animals in the remote segments of the alley during the middle and last blocks of extinction trials. On the last five-trial block, the curves for the two groups crossed, with the drugged animals running more slowly in the earlier segments and faster in the more remote ones. In separate analyses of the data from the second and last blocks of trials, the interactions of Treatments by Segments proved to be significant $[F(7,56)=2.27, p<.05$, and $F(7,56)=3.34, p<$ .01 , respectively].

In the second experiment, a similar three-way analysis over all 15 extinction trials disclosed a significant drug effect $[\mathrm{F}(1,8)=6.35, \mathrm{p}<.05]$, with the ethanol speed gradient higher than that obtained under saline. Seemingly, alcohol augmented the running speeds of the fear-motivated animals during the later blocks of extinction trials. In this analysis, the Segments by Treatments interaction was not significant $(F<1.0)$, but in the second and third trial blocks, the alcohol gradient was higher and slightly flatter than the saline one. For the second trial block, $F(1,8)=6.65, \mathrm{p}<.05$, for the treatment effect and $F(7,56)=20.2, p<.01$, for the Treatments by Segments interaction. Comparable values for 
the last five-trial blocks were: $\mathrm{F}(1,8)=5.31, \mathrm{p}<.05$, and $F(7,56)=16.0, p<.01$, respectively.

\section{Retracing Following Avoidance}

In these avoidance-gradient studies, the animals remained in the alley on shock-free test trials for $20 \mathrm{sec}$ and sometimes had time, after reaching the far (safe) end, to turn around and move back toward the punishment zone. In Experiment 1, rats that had been injected with ethanol ran back farther than did the controls, but the difference fell short of statistical significance. In Experiment 2, the difference again favored the ethanol subjects and was significant $[t(8)=2.37, p<.05]$. At first thought, these data might suggest that ethanol reduced the animals' fear of the punishment zone, as predicted by the tensionreduction theory. But these observations, it must be emphasized, were made on the same trials and on the same subjects whose isolated avoidance gradients were not significantly lowered by ethanol. Indeed, the reliably greater retracing was observed in rats whose isolated avoidance gradients were higher under alcohol than were those of nondrugged controls.

Our confidence in the generality of the ethanol retrace effects was subsequently increased by the results of a third experiment (Brown \& Mansfield, Note 2) involving a different paradigm. A withinsubject design was followed in which shock-escape training trials and avoidance test trials were administered to all of the 14 albino rats under both drug and placebo conditions. The drug was administered orally to the thirsty animals in a sugar-water vehicle, the vehicle alone serving as a control solution. Following the escape and avoidance trials, the animals were put into the alley at the end opposite to the punishment zone and were permitted to move freely back toward the shock region. On the first day, half of them were given two such test trials under ethanol (mean dose $=1.4 \mathrm{~g} / \mathrm{kg}$ ) and half were given two trials under sugar water. The drug conditions were reversed the following day. As in the second study by Brown and Lee, when the animals were tested under ethanol, they ran significantly farther back toward the punishment region than when they were tested under placebo conditions $[t(13)=2.56, p<.05]$. The mean values obtained in the Brown-Lee and Brown-Mansfield studies are shown in Figure 4.

The apparent discrepancy between our retrace and avoidance-gradient findings can be reconciled by the interference-reduction theory by assuming that only one tendency was present when the isolated gradient was being measured, but that both avoidance and approach tendencies were involved when the animals were retracing. As the rats had only limited opportunities to explore the runway prior to the test trials, hitherto latent exploratory tendencies might have
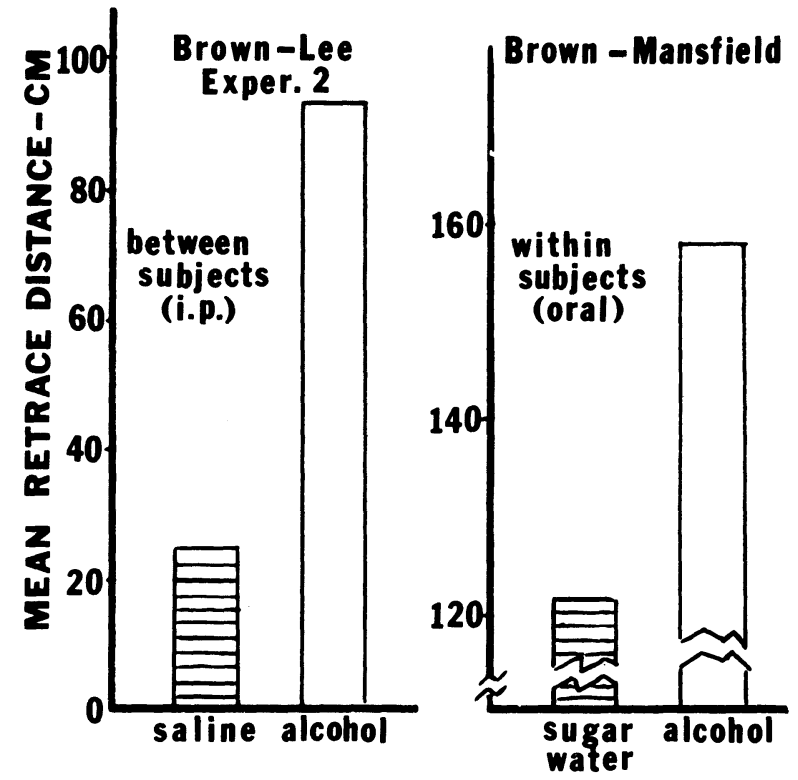

Figure 4. Results of two experiments in which shock-escape training trials were followed by shock-free avoidance trials on which the rats were allowed to retrace toward the shock region. The mean retrace distances are shown for alcohol-treated animals and for controls in a between-subjects and in a within-subjects design.

been aroused when retracing was permitted. If these tendencies were stronger, at the safe end, than the weak avoidance tendencies, the situation would qualify as an approach-avoidance conflict, with the safe end becoming bivalent. In short, on avoidance trials, the rats were at the shock end and the situation did not elicit competitive tendencies. But, when the animals turned around and ran back from the safe end, exploratory approach tendencies and weaker incompatible avoidance tendencies were evoked simultaneously. Ethanol weakened the competitive avoidance potentials still further, leaving the exploratory propensities in a dominant position. The interference-reduction theory might thus encompass both the lack of an ethanol effect on avoidance gradients alone and the presence of an effect on reapproach trials. A fear-reduction conception is consistent with the reapproach findings but not with the avoidance-gradient data.

\section{Avoidance in an Approach-Avoidance Conflict Situation}

As we have seen, both the tension-reduction and interference-reduction hypotheses lead to the prediction that inebriated animals will be more likely than sober ones to approach a bivalent goal from a remote starting place. The two interpretations yield opposite predictions, however, as to what should happen if the subjects were to be introduced into the situation at the bivalent region (as 
at the left in Figure 1) under conditions in which the avoidance is stronger there than the approach but weaker at some remote place. Fear-reduction theory suggests that after an animal has consumed ethanol it should run only as far as $I_{2}$, whereas the sober subject should move on to $\mathrm{I}_{1}$. So, on that theory, the inebriated subject should withdraw less far from the goal than should a sober subject.

The interference-reduction theory, however, leads to the expectation that when the animal is at the bivalent region, ethanol will weaken the subtractive effectiveness of the approach tendency relative to the avoidance. Consequently, the intersection of the gradients should be shifted farther away from the bivalent goal to a location at the right of $I_{1}$ in Figure 1 and the drugged animals should retreat farther from the goal than should the controls. Because the two hypotheses lead to such clearly different predictions, we have found it desirable to determine experimentally how ethanol affects goal avoidance as well as goal approach in an approach-avoidance conflict.

In each of three replications of essentially the same experiment (Brown \& Mansfield, Note 3), hungry albino rats were first trained to approach the lighted end of a $305-\mathrm{cm}$ alley for food. Half the trials were administered $15 \mathrm{~min}$ after the subjects had ingested a $3 \%$ solution of ethanol and sugar water and half following the ingestion of sugar water alone. After the approach behavior was firmly established, three shockescape trials were administered on each of 2 days, each subject receiving three such trials under ethanol and three under sugar water in counterbalanced order. On every escape trial, the rats were dropped into the lighted (goal) end of the runway where they received a .3-sec shock of approximately .7 mA from which they could immediately escape by running toward the darker end. Tests of approach (from the dark end) and of avoidance (from the lighted end) were subsequently conducted over 4 days, on each of which the rats were given two shock-free trials of 10-sec duration. One of the daily pair of trials was goal avoidance and one was goal approach. On 2 of the 4 days, the animals were responding under ethanol (mean dose $=1.7 \mathrm{~g} / \mathrm{kg}$, orally ingested), and on 2 under sugar-water placebo, in counterbalanced sequence. On every test trial, two observers recorded how far each animal moved from the starting position during the $10 \mathrm{sec}$ test.

In each of the three replications, the animals approached the bivalent goal more closely following the drinking of ethanol than after they had consumed sugar water. The grand means for the three replications combined $(n=28)$ are plotted in the upper part of Figure 5, where distances moved under sugar water and under ethanol are indicated by the appropriately labeled bars. The closer approach of the animals when under ethanol was statistically

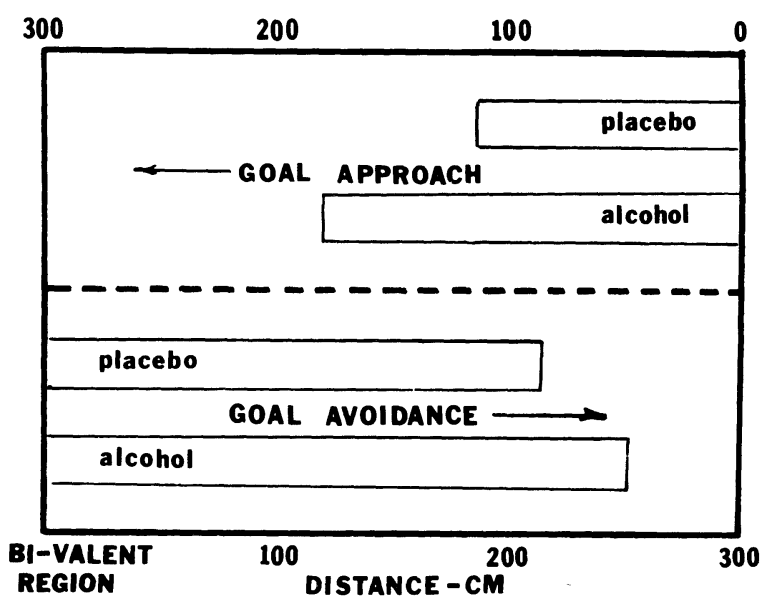

Figure 5. The bars in the upper half of the figure show that alcohol-treated rats in an approach-avoidance situation approach closer to a bivalent region than do controls when both are started some distance away from that region. The bars in the lower half reveal that ethanol also results in the animals' running farther from the bivalent region when trials involve starts at that region.

significant $[\mathrm{t}(27)=5.5, \mathrm{p}<.01]$, confirming Conger's original findings.

However, when the animals were started at the bivalent region, they retreated farther under ethanol than under placebo in all three replications. The corresponding grand means are graphed at the bottom of Figure 5. The difference favoring the drugged condition was statistically significant $[\mathrm{t}(27)$ $=2.52, \mathrm{p}<.05]$, an outcome consistent with the interference-reduction, but not with the fearreduction, conception.

A comparison of the upper and lower segments of Figure 5 reveals that the animals ran farther when retreating than when approaching, a superiority evidenced under both ethanol and placebo conditions. Since distance run may index the position of the intersection of the gradients, these data suggest that the location of the intersection is affected by direction of movement. Apparently, the intersection is shifted away from the bivalent region when the animals are pointed away from it, and toward that region when they are headed toward it.

\section{AVOIDANCE-AVOIDANCE CONFLICT AND ETHANOL}

An avoidance-avoidance conflict situation is one in which an individual has been punished at each of two spatially separated regions and has thereby acquired tendencies to avoid them both. If suitable barriers are present, conflict will be generated, since any attempt to avoid either region brings the organism closer to the other. The aversive stimuli foster the acquisition of locomotor avoidance habits and of 
classically conditioned fear. The resultant locomotor excitatory tendencies presumably take the form of gradients like those shown by solid lines in Figure 6, and the differences between their strengths, at every point, control the direction and vigor of response. Because of random variations in internal and external stimulus conditions, the heights of the gradients are expected to fluctuate over time, leading to lateral displacements of the position of the intersection and to correlated variations in the place at which movement ceases. The dashed lines in Figure 6 depict hypothetical values of such fluctuations, with $I_{2}$ and $I_{3}$ denoting the extreme positions of the intersection and $I_{1}$ representing its average location.

If tension-reduction theory were extended to the avoidance-avoidance situation (cf. Brown \& Crowell, 1974), ethanol would be expected to attenuate the conditioned fear evoked by the cues of both goal regions, and hence to lower and flatten both gradients equally. As a consequence, the distance separating $I_{2}$ and $\mathrm{I}_{3}$ should increase, widening the range of lateral movement. Additionally, the difference between the gradients at points other than the intersection (where the difference is always zero) should decrease, leading to less vigorous responding and to slower running speeds. Such a symmetrical lowering of both gradients would not, however, produce a constant shift in the mean position of the intersection.

In contrast, the interference-reduction conception would predict faster running speeds under ethanol in the avoidance-avoidance situation. This expectation stems from the assumption that when the subject is at an extreme position, at the far left, say in Figure 6, ethanol weakens the left-going tendency, which at that point is the weaker of the

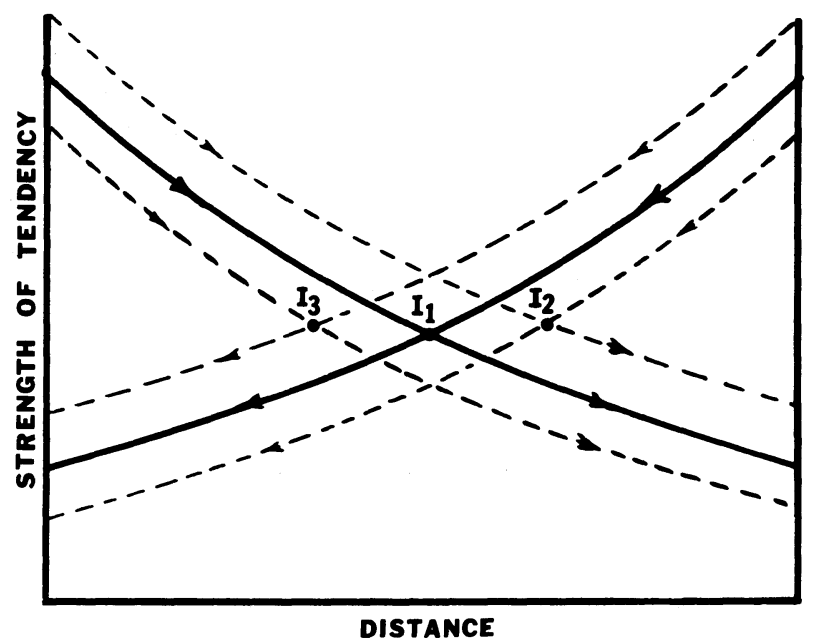

Figure 6. Hypothetical spatial gradients in the strengths of avoidance tendencies produced in a single subject by the administration of aversive stimulation at the extremes of a distance continuum. The dashed lines depict random fluctuations in the strengths of the tendencies and the possible effects thereof on the position of the gradients' intersection.

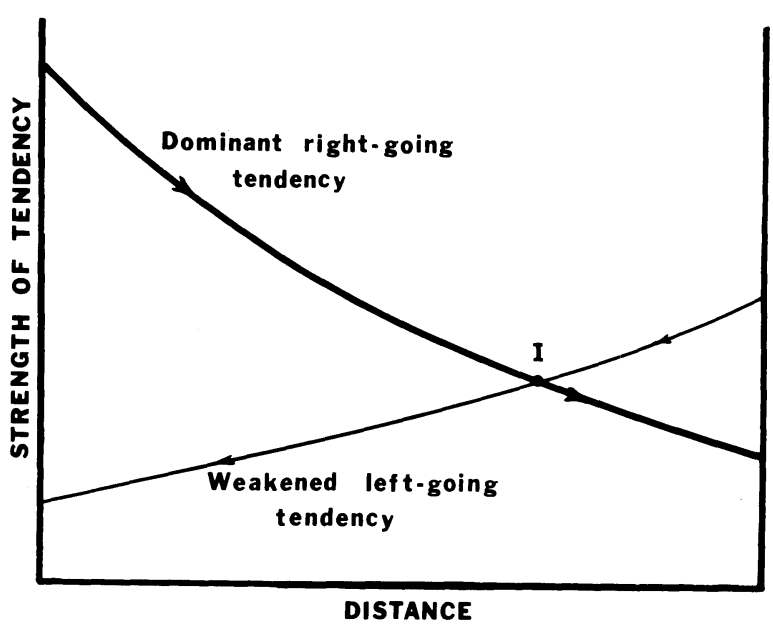

Figure 7. A possible effect of ethanol on competitive avoidance tendencies as envisioned by the interference-reduction notion. With the animal situated at the left of the diagram, the drug is assumed to weaken the subordinate left-going tendency and hence to strengthen the net right-going tendency and to shift the intersection toward the right. Increased running speeds under ethanol, rather than diminished speeds, are thereby predicted.

two, leaving the right-going tendency essentially unaffected. As a result, as Figure 7 shows, the difference between the two tendencies would be increased, not diminished, by the drug. In addition, ethanol would produce a rightward shift of the intersection. The relations appropriate to left-going trials would be represented by a mirror image of Figure 7. With the results of both kinds of trials combined, ethanol should increase the distance between the extreme positions of the intersection and widen the range of lateral movement. In brief, then, the interference-reduction conception predicts (1) faster starting and running speeds under ethanol in an avoidance-avoidance situation and (2) a wider range of lateral vacillatory movement. The theory, in its more detailed form, also predicts that the distribution of stopping points will be more distinctly bimodal under ethanol, with the mode for rightgoing trials falling to the right of center and the mode for left-going trials to the left of center.

The results of two studies that bear on these predictions are available. In the first, Mansfield, Eaton, Cunningham, and Brown (1977) trained rats, by shock-escape procedures, to withdraw from whichever end of a gray alley was made distinctive by an intermittent light-tone signal. Half the subjects were trained following the oral ingestion of an ethanolsugar-water solution and half after consuming plain sugar water. As governed by a 2 by 2 factorial design, half of each group was tested for conflict reactions under the drug $(2.4 \mathrm{~g} / \mathrm{kg})$ and half under the sugar-water control condition. On the 30 -sec, shock-free tests, the intermittent signals were presented at both ends of the runway to arouse both 
avoidance tendencies simultaneously. The rats were dropped into one or the other end of the alley, and movement was accurately recorded by means of floor-mounted strain gauges and associated equipment. The major dependent variables were: length of initial run, width of vacillation range, total backand-forth movement, and running speed.

The combined results from two replications of this procedure are shown in the upper portion of Table 1 , in which group treatment conditions and ethanol doses are listed in the first two columns, respectively, and dependent variables in the others. Length of initial run was defined as distance traversed before the first definite pause. Clearly, animals dosed with ethanol ran farther than those that had ingested sugar water alone $[F(1,36)=13.6, p<.01]$. Vacillation range indicates the maximum width of back-and-forth movements. As predicted by both theories, this was wider under ethanol than under placebo conditions $[F(1,36)=4.64, p<.05]$. Total movement was significantly greater under ethanol $[F(1,36)=27.3, p<.01]$, which is consistent with the vacillation-range results. These two measures are not, of course, necessarily correlated. Finally, the observation that running speeds were significantly faster under the drug $[\mathrm{F}(1,36)=9.53, \mathrm{p}<.01]$ is of special importance because it fits the interferencereduction but not the fear-reduction theory. On the latter view, the difference between the opposing gradients should have been reduced by the drug and speeds should have declined. But, on the former, the drug weakens the already weaker competitive tendency and augments the separation of the gradients.

In the second study of avoidance-avoidance conflict and ethanol, Mansfield (1977) confirmed and extended the findings of the first. Nine groups of rats were employed in a 3 by 3 factorial design in which ethanol concentration and dosage were varied during both avoidance training and conflict testing. The drug was combined with sugar water

Table 1

Data from Two Studies of Avoidance-Avoidance Conflict with Ethanol as the Major Independent Variable

\begin{tabular}{lrrrrr}
\hline $\begin{array}{c}\text { Testing } \\
\begin{array}{c}\text { Condition } \\
\text { Solution }\end{array}\end{array}$ & $\begin{array}{c}\text { Drug } \\
\text { Dose }\end{array}$ & $\begin{array}{c}\text { Length of } \\
\text { Initial } \\
\text { Run }\end{array}$ & $\begin{array}{c}\text { Vacil- } \\
\text { lation } \\
\text { Range }\end{array}$ & $\begin{array}{c}\text { Total } \\
\text { Move- } \\
\text { ment }\end{array}$ & $\begin{array}{c}\text { Running } \\
\text { Speed }\end{array}$ \\
\hline \multicolumn{5}{c}{ Mansfield et al. (1977) } \\
Sugar Water & .0 & 72.4 & 64.8 & 378.6 & 21.2 \\
EtOH 4.8\% & 2.4 & 125.4 & 101.7 & 526.6 & 30.4 \\
& \multicolumn{5}{c}{ Mansfield (1979) } \\
Sugar Water & .0 & 74.9 & 55.5 & 211.3 & 19.8 \\
EtOH 3\% & 1.9 & 93.5 & 82.3 & 278.3 & 33.1 \\
EtOH 6\% & 2.8 & 110.0 & 115.3 & 349.5 & 42.9 \\
\hline
\end{tabular}

Note-Distances are given in centimeters; speeds are given in centimeters per second; drug doses are given in grams per kilogram body weight. to form solutions containing $0 \%, 3 \%$, and $6 \%$ ethanol, which were administered orally to waterdeprived rats. On half the shock-escape training trials, the animals ran away from one end of a narrow black alley, and on the other half they escaped from the opposite end of a wide, white alley. Conflict tests were conducted in a medium-width alley with one white and one black wall. On each trial, the animal was dropped into the end of the alley (or near it on half the trials), after which all backand-forth movements were recorded in detail for $15 \mathrm{sec}$.

The dosages used during the test phase and the dependent variables, which were the same as in the first study, appear in the lower part of Table 1. As these entries indicate, the correspondence between the two studies was excellent, with clearly ordered dose-response relations emerging for all measures in the second investigation. Length of initial run increased with concentration $[F(2,63)=3.3, p<.05]$, vacillation range increased with concentration $[F(2,54)=10.7, p<.01]$, and so did amount of total back-and-forth movement $[\mathrm{F}(2,65)=15.3$, $\mathrm{p}<.01$ ]. Running speeds were positively related to ethanol dose, with the dose-speed relation significant $[F(2,63)=6.3, p<.01$. These data thus confirm the earlier observations of Mansfield et al. (1977) and provide further support for the interferencereduction theory as against the fear-reduction notion.

\section{DISCUSSION}

An interference-reduction interpretation of the effects of alcohol on conflict behavior has been proposed here as an alternative to the classical tensionreduction conception. We have suggested, in essence, that the drug weakens the competitive potency of the lesser of any two simultaneously instigated incompatible reaction tendencies. Since response strength is assumed to vary directly with the difference between the strengths of dominant and subordinate tendencies, the drug's main effect is expected to be enhancement of the behavioral efficacy of the dominant tendency. Ethanol should have little effect on performance in situations in which only one excitatory tendency is present or in which the dominant one is far stronger than its competitors. In the first case, no competitor is present to be weakened, and in the second, the competitor, though present, is so weak that further weakening by the drug would be of no consequence. Ethanol should also have no effect when competitive tendencies are of equal average strengths, provided random departures from equality are small and/or short-lived.

When the weaker tendency is inhibitory, our interference-reduction conception corresponds to Pavlov's (1927) assertion that ethanol selectively 
attenuates inhibition. On the present view, however, ethanol does not degrade inhibitory tendencies alone; it weakens whichever of two competitive tendencies is the weaker, whether inhibitory or excitatory. There is a sense, of course, in which the weaker of any two excitatory tendencies functions as an "inhibitor" of the stronger. It is these "functional inhibitors," these action-constraining processes, that are seen here as uniquely sensitive to the debilitating effect of the drug. Our hypothesis departs from tradition in noting that ethanol can have a disinhibiting-like effect even when the reaction tendencies are both excitatory.

The tension-reduction hypothesis holds that ethanol affects behavior primarily by reducing feargenerated motivation. The interference-reduction view involves no specific assumptions as to the mechanisms, whether motivational or associative, or both, that might be involved. One such possibility is that alcohol disrupts whichever central systems are normally responsible for the integration of competing reaction potentials. Another is that it elevates sensory or reaction thresholds, thereby degrading the effectiveness, in competition, of weaker tendencies. Still another is that the drug interferes with the organism's ability to attend simultaneously to sets of cues demanding incompatible behaviors, so that only the more salient ones are effectively processed (Moskowitz \& DePry, 1968).

As presently formulated, the interference-reduction theory does not address the question of how competition-produced emotional tension will itself be affected by the drug. As Brown and Crowell (1974) have noted, if the intensity of such tension varies directly with the absolute strengths of the competing tendencies and inversely with the difference between them, drug-induced shifts in the heights and/or slopes of the gradients will either increase or decrease that tension, depending on a variety of boundary conditions.

The experimental data reported here support the interference-reduction view in cases in which its predictions differ from those of the fear-reduction hypothesis. For one, alcohol did not reduce the heights of isolated avoidance gradients as predicted by the fear-reduction notion. For another, when rats were placed into a bivalent region where the tendency to avoid was stronger than that to approach, they retreated farther under ethanol than under control conditions. This finding, too, conflicts with the classic theory. Finally, compelling evidence has been presented to show that in an avoidanceavoidance conflict task, locomotor speeds on nonshock test trials do not decrease under ethanol; instead, they increase.

Although the paradigms used in our studies were deliberately chosen to produce incompatible reactions, there are many others, such as two-way shuttle avoidance, extinction, passive avoidance, Sidman avoidance, DRL, and open-field exploration, that may also involve competing tendencies. An extensive review of the vast literature that might illuminate the applicability of our proposal to these cases is obviously beyond the scope of this paper. Still, brief discussions of the first two (two-way shuttle avoidance and extinction) may be of value.

The two-way shuttle task resembles the avoidanceavoidance conflict problem in that the subjects are shocked at each of two spatially separated regions and must move repeatedly, on signal, to a place where shock has recently been administered. Presumably, the tendency to leave the compartment in which the animal is temporarily located will be strengthened, prior to shock onset, by the CS, increasing the probability of a successful avoidance reaction. On the fear-reduction theory, ethanol should weaken the acquired fear drive elicited by the apparatus cues and the CS, thereby reducing the difference between the competing avoidance tendencies and degrading shuttlebox performance. The interference reduction view, however, implies that ethanol should selectively weaken any subordinate interfering tendency and, by thus amplifying the difference between the opposing tendencies, enhance performance.

Experiments bearing directly on these predictions are relatively few in number, but, in two, moderate doses of ethanol facilitated two-way shuttle responding (Chesher, 1974; Scobie \& Bliss, 1974). Wallgren and Savolainen (1962) found that doses below $2 \mathrm{~g} / \mathrm{kg}$ had no effect on shuttle reaction times to shock signals and higher doses depressed performance. Crow (1966) also found that moderate doses $(1.9 \mathrm{~g} / \mathrm{kg})$ during the acquisition of shuttle responding had no effect on acquisition rate, but that relearning after a 48-h interval was faster for alcoholtreated rats. Broadhurst and Wallgren (1964) have reported that intertrial crossings are increased by ethanol, although the drug had no effect on the frequency of conditioned avoidance responses. Increases in intertrial crossings due to the drug are consistent with expectations from an interferencereduction notion, as is DiGiusto and Bond's (1977) observation of increases in pseudoconditioned shuttle responses.

The interference-reduction theory suggests that ethanol should increase the resistance to extinction of both appetitively and aversively motivated reactions. On the premise that nonreinforcement leads in both cases to the incrementing of tendencies (inhibitory and/or excitatory) that are antagonistic to the response undergoing extinction, it would follow that, during early nonreinforced trials at least, these competitive tendencies, being weaker, 
would be differently degraded by the drug. As a consequence, the dominant response would maintain its position of superiority for a longer time.

The literature on extinction under ethanol is generally supportive of this expected result. As Mello (1968) has observed, in the majority of studies in which the drug has been administered during extinction, the resistance to extinction of fear-motivated behaviors has been increased. Baum $(1969,1970$, 1971), for example, found that moderate doses of the drug prolonged the extinction of a jump-up avoidance response in both rats and mice. Barry, Wagner, and Miller (1962) and Nelson and Wollen (1965) have observed that alcohol retards the extinction of an appetitively motivated response, and Skurdal, Eckardt, and Brown (1975) found that the extinction of a one-way avoidance response in a straight alley was retarded by the drug. Additionally, in the two experiments by Brown and Lee (Note 1), running speeds during the last of 15 nonshock trials were faster for alcohol-treated than for control animals. A study by Pawlowski, Denenberg, and Zarrow (1961), however, provided limited support for the conclusion, consistent with fear-reduction theory, that the drug accelerates the extinction of shockmotivated responses.

\section{REFERENCE NOTES}

1. Brown, J. S., \& Lee, C. Effects of ethanol on spatial locomotor avoidance gradients. Unpublished study, 1976.

2. Brown, J. S., \& Mansfield, J. G. Reapproach following avoidance as affected by alcohol. Unpublished study, 1978.

3. Brown, J. S., \& Mansfield, J. G. Avoidance of a bivalent region under ethanol in rats. Unpublished study, 1979.

\section{REFERENCES}

Barry, H., III, \& Miller, N. E. Effects of drugs on approachavoidance conflict tested repeatedly by means of a "telescope alley." Journal of Comparative and Physiological Psychology, 1962, 55, 201-210.

Barry, H., III, \& Miller, N. E. Comparison of drug effects on approach, avoidance, and escape motivation. Journal of Comparative and Physiological Psychology, 1965, 59, 18-24.

Barry, H., III, Wagner, A. R., \& Miller, N. E. Effects of alcohol and amobarbital on performance inhibited by experimental extinction. Journal of Comparative and Physiological Psychology, 1962, 55, 464-468.

BAUM, M. Paradoxical effect of alcohol on the resistance to extinction of an avoidance response in rats. Journal of Comparative and Physiological Psychology, 1969, 69, 238-240.

BAUM, M. Effect of alcohol on the acquisition and resistance-toextinction of avoidance responses in rats. Psychological Reports, 1970, 26, 759-765.

BAUM, M. Effect of alcohol on the resistance-to-extinction of an avoidance response: Replication in mice. Physiology \& Behavior, 1971, 6, 307-309.

Broadhurst, P. L., \& Wallgren, H. Ethanol and the acquisition of a conditioned avoidance response in selected strains of rats. Quarterly Journal of Studies on Alcohol, 1964, 25, 476-489.
Brown, J. S. Principles of intrapersonal conflict. Journal of Conflict Resolution, 1957, 1, 135-154.

Brown, J. S., \& Crowell, C. R. Alcohol and conflict resolution: A theoretical analysis. Quarterly Journal of Studies on Alcohol, $1974,35,66-85$.

Cappell, H., \& Herman, P. Alcohol and tension reduction: A review. Quarterly Journal of Studies on Alcohol, 1972, 33, 33-64.

Chesher, C. B. Facilitation of avoidance acquisition in the rat by ethanol and its abolition by $\alpha$-methyl p-tyrosine. Psychopharmacologia, 1974, 39, 87-95.

Chittel, S. M., \& Sheth, U. K. Effect of drugs on conditioned avoidance response in rats. Archives of International Pharmacodynamics, 1963, 144, 471-480.

Conger, J. J. The effects of alcohol on conflict behavior in the albino rat. Quarterly Journal of Studies on Alcohol, 1951, 12, $1-29$.

Crow, L. T. Effects of alcohol on conditioned avoidance responding. Physiology \& Behavior, 1966, 1, 89-91.

DiGiusto, E. L., \& Bond, N. Enhancement of pseudoconditioning and retardation of escape by low doses of ethanol. Pharmacology, Biochemistry and Behavior, 1977, 6, 175-177.

FrEed, E. X. The effect of alcohol upon approach-avoidance conflict in the white rat. Quarterly Journal of Studies on Alcohol, 1967, 28, 236-254.

Freed, E. X. Effect of alcohol on conflict behaviors. Psychological Reports, 1968, 23, 151-159. (a)

FrEED, E. X. Effect of self-intoxication upon approach-avoidance conflict in the rat. Quarterly Journal of Studies on Alcohol, 1968, 29, 323-329. (b)

Harris, H. E., Piccolino, E. B., Roback, A. B., \& Sommer, D. K. The effects of alcohol on counterconditioning of an avoidance response. Quarterly Journal of Studies on Alcohol, 1964, 25, 490-497.

Hull, C. L. The goal gradient hypothesis applied to some "field force" problems in the behavior of young children. Psychological Review, 1938, 45, 271-299.

MANSFIELD, J. G. Dose-related effects of ethanol on avoidanceavoidance conflict in the rat. Unpublished MS thesis, University of Oregon Health Sciences Center, Portland, Oregon, 1977.

Mansfield, J. G., Eaton, N. K., Cunningham, C. L., \& Brown, J. S. Ethanol and avoidance-avoidance conflict in the rat. Physiological Psychology, 1977, 5, 197-203.

MELLO, N. K. Some aspects of the behavioral pharmacology of alcohol. In D. J. Effron (Ed.), Psychopharmacology: A review of progress, 1957-1967 (U.S. Public Health Service Publication No. 1836). Washington, D.C: Government Printing Office, 1968.

Mille r, N. E. Experimental studies in conflict. In J. McV. Hunt (Ed.), Personality and the behavior disorders. New York: Ronald Press, 1944.

Miller, N. E. Effects of drugs on motivation: The value of using a variety of measures. In O. v. St. Whitelock (Ed.), Techniques for the study of behavioral effects of drugs. Annals of the New York Academy of Sciences, 1956, 65, 318-333.

Miller, N. E., \& Barry, H., III. Motivational effects of drugs: Methods which illustrate some general problems in psychopharmacology. Psychopharmacologia, 1960, 1, 169-199.

Moskowitz, H., \& Asato, H. Effect of alcohol upon the latency of responses learned with positive and negative reinforcers. Quarterly Journal of Studies on Alcohol, 1966, 27, 604-611.

Moskowitz, H., \& DEPRY, D. Differential effect of alcohol on auditory vigilance and divided-attention tasks. Quarterly Journal of Studies on Alcohol, 1968, 29, 54-63.

Nelson, P. B., \& Wollen, K. A. Effects of ethanol and partial reinforcement upon runway acquisition. Psychonomic Science, $1965,3,135-136$.

Pavlov, I. P. Conditioned reflexes. Oxford: Oxford University Press, 1927. 
Pawlowski, A. A., Dennenberg, V. H., \& Zarrow, M. X. Prolonged alcohol consumption in the rat. II. Acquisition and extinction of an escape response. Quarterly Journal of Studies on Alcohol, 1961, 22, 232-240.

REynolds, G. S., \& VAN Sommers, P. Effects of ethyl alcohol on avoidance behavior. Science, 1960, 132, 42-43.

Scoвie, S. R., \& Bliss, D. K. Ethyl alcohol: Relationships to memory for aversive learning in goldfish (Carassius auratus). Journal of Comparative and Physiological Psychology, 1974, 86, 867-874.

Skurdal, A. J., Eckardt, M. J., \& Brown, J. S. The effects of alcohol on escape learning and on regular and punished extinction in a self-punitive situation with rats. Physiological Psychology, 1975, 3, 29-34.

SmART, R. G. Effects of alcohol on conflict and avoidance behavior. Quarterly Journal of Studies on Alcohol, 1965, 26, 187-205.

SomiYa, T. Adient reaction potential and abient reaction potential as a function of distance from the object. (I). Japanese Journal of Psychology, 1960, 31, 26-34.

Wallgren, H., \& Savolainen, S. The effect of ethyl alcohol on conditioned avoidance response in rats. Acta Pharmacologica et Toxicologica, 1962, 19, 59-67.

(Received for publication January 2, 1980; revision accepted May 5, 1980.) 